\title{
Perfil sociodemográfico, socio-culturales y académicos de estudiantes de enfermería en una institución de educación privada
}

Profile sociodemographic, sociocultural and academic of nursing students in an institution of education private

Perfil sociodemográfico, sociocultural e acadêmico de estudantes de enfermagem em uma instituição privada de ensino

Thaís Araújo da Silva ${ }^{1}$, Genival Fernandes de Freitas ${ }^{2}$

\begin{abstract}
${ }^{1}$ Enfermeira. Doutoranda do Programa de Pós-Graduação em Gerenciamento em Enfermagem da EEUSP.
${ }^{2}$ Livre Docente, Departamento de Orientação Profissional da Escola de Enfermagem da USP. Líder do Grupo de Pesquisa História e Legislação da Enfermagem (EEUSP)

Cómo citar este artículo en edición digital: Silva, T.A., Freitas, G.F. (2018). Perfil sociodemográfico, socio-culturales y académicos de estudiantes de enfermería en una institución de educación privada. Cultura de los Cuidados (Edición digital), 22(52). Recuperado de http://dx.doi.org/10.14198/cuid.2018.52.12

Endereço: Thais Araújo da Silva Rua Antônio Arnaldo Albergaria Pereira, 1060 - CEP 13065-010 Campinas. São Paulo - SP. Brasil

Correo electrónico: taarsi2@hotmail.com Recibido: 13/03/2018; Aceptado: 21/06/2018
\end{abstract}

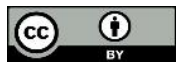

\section{ABSTRACT}

This article aims to map and discuss the sociodemographic, sociocultural and academic profile of one ordinary student enrolled in a Nursing course linked to a Private High Education Institution in São Paulo city, Brazil. An exploratorydescriptive study was done, using a simple and not inferential statistic. It was revealed that the majority were female, originally from white ethnicity, married, belonging to evangelical groups, aged between 31 and 40 years; most of them have predominantly about four members in it's families; the family income is about one thousand and three thousand reais and the majority have economic dependence. The majority group was graduated in elementary/middle level in public schools; had a student scholarship during graduation; the largest number decided to choose nursing as the first choice. Most of them were brazilians; concentrated in the southeast region of the country and comes from the eastern region of São Paulo state; $26 \%$ used to watch television as the main leisure activity. This study may become an important tool in the restructuring of actions that address the demands of students and the nursing profession.

Keywords: Students, nursing, Cultural characteristics, Social class. 


\section{Cultura de los Cuidados}

\section{RESUMO}

Este artigo tem como objetivo mapear e discutir o perfil sociodemográfico, sociocultural e acadêmico do aluno matriculado em um curso de Enfermagem ligado a uma Instituição de Ensino Superior privada no município de São Paulo, Brasil. Foi feito um estudo exploratório-descritivo, utilizando-se de uma estatística simples e não inferencial. Revelou-se que a maioria é do sexo feminino, de etnia branca, casada, evangélica, com idade entre 31 e 40 anos de idade; grande parte possui quatro integrantes na família; renda familiar entre mil e três mil reais e tem dependente econômico; formou-se no ensino fundamental/médio em escolas públicas; possuía bolsa estudantil durante a graduação; optou pela Enfermagem como primeira escolha. Grande parte é brasileira; concentra-se na região Sudeste do país e provém da região Leste do Estado de São Paulo; 26\% assistem televisão como a principal atividade de lazer. Este estudo poderá tornar-se uma ferramenta importante na reestruturação das ações que contemplem as demandas dos estudantes e da profissão Enfermagem.

Palavras-chave: Estudantes de enfermagem, Ambiente sociocultural, Ambiente socioeconômico.

\section{RESUMEN}

Este artículo tiene como objetivo organizar y discutir el perfil sociodemográfico, sociocultural y académico del estudiante inscrito en un curso de enfermería conectado a una institución de educación superior privada en la ciudad de Sao Paulo, Brasil. Se trata de investigación exploratoria y descriptiva, utilizándose una estadística simple y no inferencial. Se puso de manifiesto que la mayoría son de sexo femenino, raza blanca, casado, evangélica, con edades comprendidas entre 31 y 40 años de edad; muchos tienen cuatro miembros de la familia; ingresos familiares de entre uno y tres mil reales $y$ tiene dependientes económicos. La mayoría frecuentó la enseñanza primaria/secundaria en las escuelas públicas; en enfermería, mucho tenía beca de apoyo al estudiante, durante la graduación. El número más grande optó por la enfermería como una primera opción. Mucho es de Brasil; concentrada en la región del sureste del país y proviene de la región oriental del Estado de São Paulo; 26\% ven la televisión como la actividad principal de ocio. Este estudio puede llegar a ser una herramienta importante en la reestructuración de las acciones que responden a las demandas de los estudiantes y la profesión de enfermería.

Palabras clave: Estudiantes de enfermeira, Características culturales, Clase social.

\section{INTRODUÇÃO}

No período entre 2001 e 2010 o Plano Nacional de Educação (PNE) apresentou uma política de Estado, com o objetivo de aumentar o número de Instituições Educacionais Superiores (IES) no Brasil (Brasil, 2001). Essa política adveio em meio a lutas e exigências realizadas pela sociedade civil ao mercado, para que fosse facilitado o acesso ao ensino superior (Teixeira et al., 2013). Uma consulta realizada no site do Ministério da Educação, no corrente ano, aponta que existem 39 IES no município de São Paulo que ofertam o curso de Enfermagem, sendo duas públicas e 37 privadas (Brasil, 2017). Salienta-se que o desenfreado crescimento das faculdades privadas não oferece adequada qualidade no ensino, uma vez que esse crescimento pode não acompanhar a formação de 
docentes para darem conta das tarefas (Cunha, 2004). Ademais, o mercado de trabalho na área da saúde vem se modificando e provocando transformações sociais, tecnológicas, econômicas e políticas; por isso, é imprescindível que as IES acompanhem esse ritmo acelerado e transmutável para reorganizar as estruturas educacionais (Wetterich, Melo, 2007).

A problemática do perfil dos profissionais de enfermagem formados em atuação no mercado tem sido uma tônica cada vez mais presente, tanto por parte dos órgãos que fiscalizam o exercício profissional (Conselhos), como por parte de outras organizações ou entidades (Associações de Especialistas em Enfermagem) e à própria Associação Brasileira de Enfermagem (ABEn). Essa última, a duras penas, vem lutando há décadas para assegurar a qualidade do ensino e da formação, inclusive permanente, dos profissionais que atuam no campo da enfermagem. Nesse sentido, trazendo luz a essas reflexões, despertou-se o interesse em conhecer o perfil sociodemográfico, sociocultural e acadêmico dos graduandos de Enfermagem de uma faculdade privada, localizada no município de São Paulo, a qual se insere no contexto do mercado atual. A priori, foi realizado levantamento sobre "estudantes de enfermagem" e "perfil sociodemográfico", nos anos de 2009, 2010, 2011 e 2015, na Biblioteca Virtual em Saúde (BVS). Foram levantados nove artigos, sendo encontrados apenas dois que abordavam a temática do perfil dos estudantes de enfermagem nas universidades: dentre esses, um estudo de Bublitz et al. (2015), objetivou descrever as características sociodemográficas e acadêmicas dos discentes de Enfermagem de quatro IES brasileiras, entre o período de abril de 2011 e março de 2012, por meio de um formulário. Os resultados mostraram que 705 indivíduos eram estudantes com idade superior a 18 anos, a maioria era solteira, do sexo feminino, possuía uma atividade de lazer, não praticava esporte, residia com familiares, não tinha filhos, estava satisfeita com o curso que escolhera, não recebia bolsa acadêmica e não participava de grupo de pesquisa. $\mathrm{O}$ segundo estudo realizado por Brito et al., (2009), objetivou caracterizar o perfil sociodemográfico de discentes dos cursos de Enfermagem de Belo Horizonte, MG. Para tanto, foi aplicado um questionário, em cinco IES privadas. Os resultados apresentaram que quase metade dos estudantes já atuava na área da Enfermagem, que a maioria provinha de escola pública, era solteira, com predominância do sexo feminino e apresentava idade entre 20 e 24 anos.

Com base na problemática apontada e nesse breve rapport, a presente investigação teve como objetivo mapear e discutir o perfil sociodemográfico, sociocultural e acadêmico do aluno matriculado em um curso de Enfermagem ligado a uma IES privada, no município de São Paulo, referente ao ano de 2014.

\section{METODOLOGIA}

Estudo de natureza exploratóriadescritiva, utilizando-se de uma estatística simples, descritiva e não inferencial. Utilizou-se questionário com perguntas abertas e fechadas, dirigidas aos graduandos de enfermagem da IES escolhida, o qual foi aplicado no período de 25 de outubro e 25 de novembro de 2014. Como critérios de inclusão dos participantes, definiram-se os seguintes pontos: graduandos de diferentes semestres (1..$^{\circ}$ ao $\left.9 .^{\circ}\right)$ e que, ao serem convidados, 
aceitaram de forma livre e espontânea participar como sujeitos da investigação. Justifica-se a escolha por diferentes semestres, tendo em vista a necessidade de ampliar quantitativamente $\mathrm{o}$ retrato da amostra e caracterizar, com melhor fidedignidade, o perfil do alunado da referida instituição. A amostra se configurou com o contingente de 300 graduandos. A coleta dos dados foi realizada por um dos pesquisadores, o que ocorreu diretamente do contato desse com os participantes, após explicar-lhes os objetivos da pesquisa e apresentar-lhes o Termo de Consentimento Livre e Esclarecido (TCLE). Tal participação ocorreu em ambiente de sala de aula, em dias e horários previamente agendados com os graduandos e docentes da formação, além do prévio consentimento da coordenação do curso.

Para a descrição dos perfis dos participantes dessa pesquisa, foi elaborado um banco de dados no Microsoft Excel, no qual possibilitou analisar os perfis por meio de frequências simples das seguintes variáveis: sexo, etnia, religião, faixa etária, estado civil, quantitativo de pessoas que integram a família, caracterização do responsável pela família, renda familiar, dependente econômico, quantitativo de pessoas que trabalham na família, modelo estudantil no ensino fundamental e médio, bolsa estudantil e o tipo de bolsa, escolha profissional, caracterização dos discentes que trabalham como auxiliar ou técnico de enfermagem e há quanto tempo, caracterização dos que trabalham em outras áreas, nacionalidade, procedência das regiões do Brasil, região de residência (no Estado de São Paulo) e atividades de lazer.

Pontua-se que este estudo é oriundo da dissertação de mestrado intitulada
"Identidade e escolhas profissionais na perspectiva de graduando de Enfermagem", a qual aprovada pelo Comitê de Ética em Pesquisa da Escola de Enfermagem da Universidade de São Paulo (CAE: 31704214.5.0000.5392) e, também, pela coordenação da IES pesquisada, observando a recomendação da Resolução 466/12 do Conselho Nacional de Saúde. Todos os participantes do estudo assinaram o Termo de Consentimento Livre e Esclarecido, de forma voluntária, após esclarecimento da proposta da investigação. Foi assegurado o anonimato, a privacidade e o sigilo absoluto das informações.

\section{RESULTADOS}

A amostra de 300 participantes representou cerca de $30 \%$ do total de graduandos matriculados no período naquela IES. Quanto às características socioculturais dos discentes estudados, observa-se na Tabela 1, o predomínio do sexo feminino ( $82 \%$ ); a maioria se considera de etnia branca (35\%); denota-se predominância da religião evangélica, em $40 \%$; $26 \%$ se identificaram como católicos; faixa etária entre 31 e 40 anos e a maioria é casada (38\%).

Na Tabela 2 a maioria dos discentes é casada, o que corresponde a $38 \% ; 26 \%$ possuem quatro integrantes na família; sendo que o responsável pela família é o cônjuge, perfazendo um percentual de 31\% e de $30 \%$ a própria pessoa; a maior evidência $(40 \%)$ da renda familiar está entre mil e três mil reais; $60 \%$ têm pelo menos um dependente econômico; $46 \%$ dos participantes relataram que pelo menos duas pessoas da família trabalham. 
TABELA 1: Distribuição de discentes segundo sexo, etnia, religião e idade. São Paulo, 2014

\begin{tabular}{llc}
\hline & Variável & $\%$ \\
\hline Sexo & & \\
& Feminino & $82,00 \%$ \\
& Masculino & $18,00 \%$ \\
& & $\mathbf{1 0 0 , 0 0 \%}$ \\
\hline
\end{tabular}

\section{Etnia}

Branca

$35,00 \%$

Negra

$33,00 \%$

Parda

$25,00 \%$

Amarela

$3,00 \%$

N.I

$4,00 \%$

Total

$100,00 \%$

\section{Religião}

Evangélica

$40,00 \%$

Católica

$26,00 \%$

Espírita

$13,00 \%$

Budista

$2,00 \%$

N.I

$19,00 \%$

Total

$100,00 \%$

\section{Faixa Etária}

$$
\begin{aligned}
& 0-20 \\
& 21-30 \\
& 31-40 \\
& 41-50 \\
& \text { N.I }
\end{aligned}
$$$$
1,00 \%
$$$$
26,00 \%
$$$$
38,00 \%
$$$$
21,00 \%
$$$$
14,00 \%
$$

Total $100,00 \%$ 
TABELA 2: Distribuição dos discentes quanto ao estado civil, quantitativo de integrantes da família, responsável pela família, renda familiar, dependente econômico e quantitativo de pessoas que trabalham na família. São Paulo, 2014

\begin{tabular}{|c|c|c|}
\hline & Variável & $\%$ \\
\hline \multicolumn{3}{|l|}{ Estado Civil } \\
\hline & Solteiro & $31,00 \%$ \\
\hline & Casado & $38,00 \%$ \\
\hline & Divorciado & $17,00 \%$ \\
\hline & Viúvo & $5,00 \%$ \\
\hline & N.I & $9,00 \%$ \\
\hline Total & & $100,00 \%$ \\
\hline \multicolumn{3}{|c|}{ Quantas Pessoas Integram a Família } \\
\hline & 1 & $8,00 \%$ \\
\hline & 2 & $16,00 \%$ \\
\hline & 3 & $23,00 \%$ \\
\hline & 4 & $26,00 \%$ \\
\hline & $\geq 5$ & $9,00 \%$ \\
\hline & N.I & $18,00 \%$ \\
\hline Total & & $100,00 \%$ \\
\hline \multicolumn{3}{|c|}{ Responsável Pela Família } \\
\hline & Pais & $22,00 \%$ \\
\hline & Própria Pessoa & $30,00 \%$ \\
\hline & Avós & $3,00 \%$ \\
\hline & Cônjuge & $31,00 \%$ \\
\hline & N.I & $14,00 \%$ \\
\hline Total & & $100,00 \%$ \\
\hline \multicolumn{3}{|c|}{ Renda Familiar } \\
\hline & $\leq \mathrm{R} \$ 1.000,00$ & $5,00 \%$ \\
\hline & $\mathrm{R} \$ 1.001,00$ a $\mathrm{R} \$ 3.000,00$ & $40,00 \%$ \\
\hline & $>\mathrm{R} \$ 3.001,00$ & $23,00 \%$ \\
\hline & N.I & $32,00 \%$ \\
\hline Total & & $100,00 \%$ \\
\hline \multicolumn{3}{|c|}{ Dependente econômico } \\
\hline & Sim & $60,00 \%$ \\
\hline & Não & $31,00 \%$ \\
\hline & N.I & $9,00 \%$ \\
\hline Total & & $100,00 \%$ \\
\hline \multicolumn{3}{|c|}{ Quantitativo de pessoas que trabalham na família } \\
\hline & 1 & $27,00 \%$ \\
\hline & 2 & $46,00 \%$ \\
\hline & 3 & $15,00 \%$ \\
\hline & 4 & $5,00 \%$ \\
\hline & $\geq 5$ & $0,00 \%$ \\
\hline & N.I & $7,00 \%$ \\
\hline Total & & $100,00 \%$ \\
\hline
\end{tabular}


De acordo com a Tabela 3, a maioria dos discentes concluiu os níveis de formação no ensino fundamental $(74 \%)$ e médio $(80 \%)$, em escolas públicas. Ademais, 63\% possuíam, na época, algum tipo de bolsa estudantil, sendo $80 \%$ das bolsas ofertadas provenientes do Fundo de Financiamento Estudantil (Fies); $73 \%$ optaram pela Enfermagem como primeira escolha. Dos alunos trabalhadores, $56 \%$ não atuavam como auxiliar e/ou técnico de enfermagem. No entanto, desse contingente anteriormente citado, $36 \%$ atuavam em áreas como administração, vendas e/ou operacionais, ou seja, em atividades distintas da saúde. Dos 39\% que atuavam como auxiliares e/ou técnicos de enfermagem; $63 \%$ tinham mais de cinco anos nessa área.

Os dados da Tabela 4, que vem a seguir, retratam a variável: nacionalidade, na qual $96 \%$ são brasileiros. A maioria $(58 \%)$ se concentra na região Sudeste do país, sendo que boa parte $(45 \%)$ provém da região Leste do Estado de São Paulo; 26\% assistem televisão como a principal atividade de lazer.

\section{DISCUSSÃO}

Nesse estudo, verificou-se que há predomínio de discentes do sexo feminino $(82 \%)$, o que não difere, substancialmente, de outros estudos que demonstraram que esse contingente é igual ou superior a $84 \%$ (Brito et al., 2009).

Vale ressaltar que a questão de gênero na Enfermagem é amplamente discutida, pois historicamente essa profissão foi se constituindo como uma prática eminentemente feminina, pelo fato do cuidado estar relacionado às características femininas (Passos, 1996).
Por outro lado, dados de uma pesquisa sobre o perfil dos profissionais de enfermagem brasileiros, destacaram que o maior contingente de profissionais da Enfermagem é do sexo feminino (84,6\%) e apenas $15 \%$ são do sexo masculino (Conselho Federal de Enfermagem, 2015 e Fundação Oswaldo Cruz; 2015).

Corroborando essa discussão, cabe destacar estudo realizado sobre a presença de homens no curso de Enfermagem de uma das mais tradicionais escolas de Enfermagem do país, ao qual foram levantadas fichas dos ex-alunos, junto ao Serviço de Graduação da Escola de Enfermagem da Universidade de São Paulo, no período de 1950 a 1990. Esse estudo possibilitou caracterizar o perfil sociodemográfico de homens egressos da instituição estudada. $\mathrm{O}$ trabalho destaca que o quantitativo de homens que estudavam Enfermagem era muito baixo em relação ao sexo feminino (Souza Costa, Freitas, 2009).

No estudo presente, identificou-se um percentual de $35 \%$ nos discentes que se consideraram de etnia branca, o que é permitido discutir o fato de que a miscigenação é um fenômeno presente na sociedade brasileira, desde os primórdios da colonização. Esse elemento nos permite, ainda, discutir outro ponto no que tange à classificação da etnia das pessoas, consideradas negras ou brancas (Sacramento, Nascimento, 2011).

No bojo da discussão, um estudo analisou a presença da mulher negra na enfermagem profissional brasileira, trazendo à tona a discriminação racial como fenômeno marcante na sociedade e, ao mesmo tempo, fomentando debates sobre as ações afirmativas que garantam a igualdade de gênero, classe e etnia. 
TABELA 3: Distribuição dos discentes quanto ao ensino fundamental e médio; bolsa estudantil e o tipo da bolsa; escolha pela Enfermagem; profissionais atuantes na enfermagem, tempo de atuação profissional na enfermagem e em outras áreas. São Paulo, 2014

\begin{tabular}{|c|c|c|}
\hline \multicolumn{2}{|c|}{ Variável } & $\%$ \\
\hline \multicolumn{3}{|c|}{ Ensino Fundamental } \\
\hline & $\begin{array}{l}\text { Pública } \\
\text { Privada } \\
\text { N.I }\end{array}$ & $\begin{array}{l}74,00 \% \\
15,00 \% \\
11,00 \%\end{array}$ \\
\hline Total & & $100,00 \%$ \\
\hline \multicolumn{3}{|c|}{ Ensino Médio } \\
\hline & $\begin{array}{l}\text { Pública } \\
\text { Privada } \\
\text { N.I }\end{array}$ & $\begin{array}{c}80,00 \% \\
11,00 \% \\
9,00 \%\end{array}$ \\
\hline Total & & $100,00 \%$ \\
\hline \multicolumn{3}{|c|}{ Bolsa Estudantil } \\
\hline & $\begin{array}{l}\text { Sim } \\
\text { Não } \\
\text { N.I }\end{array}$ & $\begin{array}{c}63,00 \% \\
32,00 \% \\
5,00 \%\end{array}$ \\
\hline Total & & $100,00 \%$ \\
\hline \multicolumn{3}{|c|}{ Tipo de bolsa estudantil } \\
\hline & $\begin{array}{l}\text { Fies } \\
\text { Prouni } \\
\text { Outras } \\
\text { N.I }\end{array}$ & $\begin{array}{c}80,00 \% \\
13,00 \% \\
2,00 \% \\
6,00 \%\end{array}$ \\
\hline Total & & $100,00 \%$ \\
\hline \multicolumn{3}{|c|}{ A Enfermagem foi } \\
\hline & $\begin{array}{l}\text { 1. }{ }^{\mathrm{a}} \text { opção } \\
\text { 2. }{ }^{\mathrm{a}} \text { opção } \\
3 \text {. }{ }^{\mathrm{a}} \text { opção } \\
\text { N.I }\end{array}$ & $\begin{array}{c}73,00 \% \\
16,00 \% \\
6,00 \% \\
5,00 \%\end{array}$ \\
\hline Total & & $100,00 \%$ \\
\hline \multicolumn{3}{|c|}{ Trabalham como aux/téc de enfermagem } \\
\hline & $\begin{array}{l}\text { Sim } \\
\text { Não } \\
\text { N.I }\end{array}$ & $\begin{array}{c}39,00 \% \\
56,00 \% \\
5,00 \%\end{array}$ \\
\hline Total & & $100,00 \%$ \\
\hline \multicolumn{3}{|c|}{ Há quanto tempo trabalham na enfermagem } \\
\hline & $\begin{array}{l}<5 \text { anos } \\
>5 \text { anos } \\
\text { N.I }\end{array}$ & $\begin{array}{c}34,00 \% \\
63,00 \% \\
3,00 \%\end{array}$ \\
\hline Total & & $100,00 \%$ \\
\hline \multicolumn{3}{|c|}{ Trabalham em outras áreas } \\
\hline & $\begin{array}{l}\text { Administrativo } \\
\text { Vendas } \\
\text { Operacional } \\
\text { N.I }\end{array}$ & $\begin{array}{c}20,00 \% \\
11,00 \% \\
5,00 \% \\
20,00 \%\end{array}$ \\
\hline Total & & $56,00 \%$ \\
\hline
\end{tabular}


TABELA 4: Distribuição dos discentes quanto à nacionalidade; regiões de procedência; locais de residência na Grande São Paulo e atividade de lazer. São Paulo, 2014

\begin{tabular}{|c|c|c|}
\hline \multicolumn{2}{|c|}{ Variável } & $\%$ \\
\hline \multicolumn{3}{|c|}{ Nacionalidade } \\
\hline & Brasileiro & $96,00 \%$ \\
\hline & Estrangeiro & $1,00 \%$ \\
\hline & N.I & $3,00 \%$ \\
\hline \multicolumn{2}{|l|}{ Total } & $100,00 \%$ \\
\hline \multicolumn{3}{|c|}{ Procedência dentro do país } \\
\hline & Sul & $5,00 \%$ \\
\hline & Sudeste & $58,00 \%$ \\
\hline & Centro-Oeste & $3,00 \%$ \\
\hline & Nordeste & $11,00 \%$ \\
\hline & N.I & $23,00 \%$ \\
\hline \multicolumn{2}{|l|}{ Total } & $100,00 \%$ \\
\hline \multicolumn{3}{|c|}{ Região onde reside dentro do Estado de São Paulo } \\
\hline & Sul & $8,00 \%$ \\
\hline & Norte & $13,00 \%$ \\
\hline & Oeste & $5,00 \%$ \\
\hline & Leste & $45,00 \%$ \\
\hline & Grande São Paulo & $15,00 \%$ \\
\hline & N.I & $14,00 \%$ \\
\hline \multicolumn{2}{|l|}{ Total } & $100,00 \%$ \\
\hline \multicolumn{3}{|c|}{ Atividade de lazer } \\
\hline & Esporte & $9,00 \%$ \\
\hline & Passeio & $12,00 \%$ \\
\hline & $\mathrm{TV}$ & $26,00 \%$ \\
\hline & Cinema & $12,00 \%$ \\
\hline & Outros & $18,00 \%$ \\
\hline & N.I & $34,00 \%$ \\
\hline Total & & $100,00 \%$ \\
\hline
\end{tabular}


Em se tratando de religião, a maioria dos discentes relatou pertencer ao protestantismo (40\%) e $26 \%$ se declararam católicos. Assim, vê-se que $66 \%$ estão vinculados às religiões de doutrina cristã. $\mathrm{O}$ elemento religioso é parte importante da cultura, em geral; esse dado, particularmente, chamou a atenção, haja vista o fato de que o cuidado e a religião não são isolados das civilizações, pois desde os povos mais primitivos, o cuidado tornou-se elemento fundamental para a sobrevivência humana. Nessa perspectiva, sabe-se que na História da Enfermagem não é diferente, visto que, com o advento do cristianismo, nota-se forte influência e presença religiosa nos cuidados aos doentes, pobres e abandonados, com base nos ensinamentos cristãos do amor e da caridade (Freitas et al., 2010).

Por outro lado, é possível enxergar que a inculcação ideológica nas práticas de saúde e do cuidado de enfermagem estão presentes e fundamentam-se, há muito tempo, na ideologia do amor, da caridade e, portanto, do altruísmo. Tais elementos foram arraigados no meio cristão e vincularam-se aos discursos enunciados por Florence Nightingale (como precursora da enfermagem moderna ou profissional, no século XIX), ao dizer que foi designada a atuar na enfermagem em razão da vocação ou chamado divino (Gomes et al., 2007).

Os dados apresentados acima possibilitam, ainda, discutir outro elemento importante, no tocante à faixa etária dos discentes, integrantes do presente estudo. Essas informações trouxeram um diferencial da maioria das pesquisas que discutem esse aspecto, pois, nesse estudo, a maioria deles encontrava-se na faixa etária entre 31 e 40 anos de idade, o que não é de se estranhar, pois muitos eram casados.

Comparativamente, é possível detectar que dados recentes do Censo da Educação Superior mostram que cerca da metade dos sete milhões de estudantes, no Brasil, possui idade superior a 20 anos de idade e que, desses, cerca de 600 mil têm mais de 40 anos de idade (Ristoff, 2014, p. 726). Com isso, vêse que muitas pessoas já maduras e inseridas no mercado de trabalho têm buscado novas ocupações ou capacitações profissionais.

Vale ressaltar que a ampliação do acesso à educação superior no Brasil, sobretudo, a partir do ano 2000, permitiu a inclusão de grupos historicamente excluídos, principalmente nas instituições de ensino privadas, ou seja, estudantes com mais de 24 anos, população com baixa renda e estudantes provenientes de escolas públicas (Ristoff, 2014).

As variáveis apresentadas na Tabela 2 evidenciam que no estado civil a maioria dos discentes é casada (38\%); que quatro pessoas agregam a família (26\%); que o responsável pela família é o cônjuge (31\%); que a renda familiar se concentra entre $\mathrm{R} \$ 1.001,00$ e $\mathrm{R} \$$ $3.000,00 \quad(40 \%)$; que a maioria tem dependente econômico e que apenas duas pessoas trabalham na família dos discentes (46\%). As características apresentadas nessas variáveis podem indicar que os indivíduos postergam a inserção na graduação por possuírem obrigações familiares. Verificou-se que a maioria concluiu os estudos fundamental/médio em escolas públicas $(74 \%$ e $80 \%$, respectivamente). Nesse contexto, alude-se que a muitos provêm provém de escolas públicas e optam por uma IES privada (Lima et al., 2015).

É fundamental revisar as políticas pedagógicas, no que tange aos ingressantes no ensino superior que cursaram o ensino fundamental e médio em escolas públicas, haja vista que existe grande preocupação inerente à qualidade na assistência dos 
futuros enfermeiros, uma vez que consideram preocupante a base cultural pelo cenário atual da educação nas escolas públicas no Brasil (Teixeira et al., 2006).

A maioria dos graduandos desse estudo possui bolsa estudantil (63\%), sendo o grande percentual a bolsa Fies. Esse elemento deve ser visto no contexto do Plano de Desenvolvimento da Educação (PDE), de 2007, o qual propôs ampliação do acesso ao ensino superior articulado com programas de financiamento para esse nível, destacando-se, no caso do ensino privado, os programas de financiamento do Fies e o Programa Universidade para todos (ProUni), com a concessão de bolsas parciais ou integrais para estudantes de baixa renda (Severino, 2008).

A amostra revela que $39 \%$ dos participantes da pesquisa exercem a profissão de enfermagem como auxiliares ou técnicos e que 63\% têm mais de cinco anos na área. Ressalta-se que, atualmente, há 1,6 milhões de profissionais de Enfermagem no Brasil; sendo $80 \%$ deles técnicos e auxiliares; 20\% de enfermeiros (Conselho Federal de Enfermagem, 2015 e Fundação Oswaldo Cruz, 2015).

$\mathrm{O}$ estudo apontou que $73 \%$ dos graduandos escolheram a Enfermagem como primeira opção. Nesse sentido, atentase que essa área vem ganhando espaço devido ao reconhecimento de suas atribuições e de seu papel perante a sociedade, "refletindo um novo status para a profissão, o que serve de estímulo para o interesse na inserção de novos profissionais no mercado de trabalho" (Brito et al., 2009, p. 329).

Verificou-se que a grande maioria é brasileira (96\%) e provém da região Sudeste do país (58\%). O Enade, realizado em 2013 pelos estudantes de Enfermagem, informou que "a região Sudeste apresentou o maior número de estudantes inscritos, 11.354, dos quais $10.114 \quad(89,1 \%)$ estudavam em Instituições Privadas" (Brasil, 2013, p. 26). Por outro lado, grande parte dos estudantes reside na região Leste do Estado de São Paulo (45\%). Ainda que a IES estudada se localize na região Central do Estado de São Paulo, infere-se que a escolha pelo local do estudo tenha sido motivada por conta da rede de transporte que servem à localidade.

A mostra revela, ainda, que a principal atividade de lazer se concentra em assistir TV (26\%). Nessa direção, estudos apontam que muitos indivíduos praticam o lazer dentro do próprio local em que reside (Marcelino, 1996). A propositura de políticas públicas, visando o aprimoramento profissional e a formação qualificada de novos profissionais na área da enfermagem passa, necessariamente, pelo reconhecimento das variáveis estudadas, identificando-se o perfil dos ingressantes no curso de Enfermagem. Obviamente que uma realidade social tão delimitada, como a do cenário desse estudo, não permite generalizações (mesmo porque não foi esse o intuito dessa investigação), no entanto, espera-se que essa contribuição aprofunde o nível de reflexões acerca da formação do enfermeiro, do perfil e da clientela interessada pela Enfermagem, norteando e direcionando não somente as políticas públicas relacionadas à educação, mas alavancando um patamar mais elevado de exigência de qualificação do ensino ofertado pelas IES privadas, mormente no campo da Enfermagem, no país.

\section{CONCLUSÃO}

O perfil sociodemográfico, sociocultural e acadêmico do alunado pesquisado, possibilitou conhecer melhor as características da população estudada, 
demonstrando a estrutura e arranjo espacial do público-alvo. Esse ponto constituiu-se em um elemento essencial para caracterizar a realidade dos graduandos de enfermagem a fim de compreender e relacionar esse perfil com sua natureza individual e coletiva. Por conseguinte, notou-se que houve grande diferença na idade dos discentes da IES, lócus do estudo, comparativamente com pesquisas anteriormente realizadas; dado interessante, pois outros estudos apontam similaridades inerentes à faixa etária.

Julga-se imprescindível a realização de outros estudos, que ampliem essa dimensão e investiguem realidades diferentes em campos diversos, haja vista que o conhecimento a respeito do perfil sociodemográfico e sociocultural dos estudantes de enfermagem permitem desdobrar ações em prol da formação e, sobretudo, revelam-se seus anseios e expectativas em relação à profissão, para que novas ferramentas concernentes ao projeto político pedagógico surjam de maneira a auxiliar, facilitar e promover o processo ensino-aprendizagem, estabelecido ao longo da formação acadêmica.

\section{REFERÊNCIAS}

- A Brasil (2013). Ministério da Educação. Exame Nacional de Desempenho dos Estudantes (ENADE). Enfermagem. Recuperado de http://download.inep.gov.br/educacao superior/e nade/relatorio_sintese/2013/2013_rel_enfermagem. pdf.

- Brasil. (2001). Ministério da Educação. Lei n..$^{0}$ 10.172, de 9 de janeiro de 2001. Aprova o Plano Nacional de Educação e dá outras providencias. Diário Oficial da União. Brasilia: Ministério da Educação.

- Brasil. (2017). Instituições de Educação Superior e Cursos Cadastrados. Brasilia: Ministério da Educação. Recuperado de http://emec.mec.gov.br/. - Brito, A. M. R., Brito, M. J. M., Silva, P. A. B. (2009). Perfil sociodemográfico de discentes de enfermagem de instituições de ensino superior de Belo Horizonte. Esc. Anna Nery 13(2), 328-3. Recuperado de http://www.scielo.br/pdf/ean/v13n2/v13n2a13.pdf. - Bublitz, S., Guido, L. A., Kirchhof, R. S., Neves, E. T., Lopes, L. F. (2015). Perfil sociodemográfico e acadêmico de discentes de enfermagem de quatro instituições brasileiras. Rev. Gaúcha Enferm. 36(1), 77-83. Recuperado de http://www.scielo.br/pdf/rgenf/v36n1/pt_19831447-rgenf-36-01-00077.pdf.

- Conselho Federal de Enfermagem. (2015) Pesquisa inédita traça perfil da enfermagem. Diagnóstico da profissão aponta concentração regional, tendência à masculinização, situações de desgaste profissional $e$ subsalário. Recuperado de http://www.cofen.gov.br/pesquisa-inedita-tracaperfil-da-enfermagem_31258.html.

- Cunha, L. A. (2004). Desenvolvimento desigual e combinado no ensino superior: Estado e mercado. Educ. Soc. 25(88), 795-817. Recuperado de http://www.scielo.br/pdf/es/v25n88/a08v2588.pdf.

- Freitas, G. F., Siles Gonzáles, J., Bonini, B. B., Takashi, M. H. (2010). Representaciones y práticas antes de la profesionalización en enfermería: influencia religiosa. Cultura de los cuidados: historia de la enfermería iberoamericana (pp. 77-96). Alicante: Editorial Club Universitário.

- Fundação Oswaldo Cruz. (2015). Pesquisa retrata perfil de 1,6 milhão de profissionais de enfermagem no Brasil. Recuperado de http://portal.fiocruz.br/ptbr/content/pesquisa-retrata-perfil-de-16-milhaode-profissionais-de-enfermagem-no-brasil.

- Gomes, V. L. O., Backes, V. M. S., Padilha, M. I. C. S. \& Cezar Vaz, M. R. (2007). Evolução do conhecimento científico na enfermagem: do cuidado popular à construção de teorias. Invest. educ. enferm., 25(2), 108-15. Recuperado de http://www.scielo.org.co/pdf/iee/v25n2/v25n2a10. pdf.

- Lima, C. A., Vieira, M. A., Costa, F. M., Rocha, J. F. D. \& Dias, O. V. (2015). Correlação entre perfil sociodemográfico e acadêmico e formas de ingresso na graduação em enfermagem. Rev enferm UFPE, 9(4), 7986-94. Recuperado de http://www.revista.ufpe.br/revistaenfermagem/in dex.php/revista/article/viewFile/7507/pdf_7874.

- Marcelino, N. C. (1996). Estudos do lazer: uma introdução. Campinas: Autores Associados.

- Passos, E. (2012). De anjos a mulheres: ideologias e valores na formação de Enfermeiras. 1. ${ }^{\mathrm{a}}$ ed. Salvador: EDUFBA.

- Ristoff, D. (2014). O novo perfil do campus brasileiro: uma análise do perfil socioeconômico do estudante de graduação. Avaliação, 19(3), 732-47. 
Recuperado

de

http://www.scielo.br/pdf/aval/v19n3/10.pdf.

- Sacramento, A. N., Nascimento, E. R., (2011). Racismo e saúde: representações sociais de mulheres e profissionais sobre o quesito cor/raça. Rev. esc. enferm. USP, 45(5), 1142-9. Recuperado de http://www.redalyc.org/articulo.oa? $\mathrm{id}=3610333120$ 16.

- Severino, A. J. (2008). O ensino superior brasileiro: novas configurações e velhos desafios. Educar, (31), 73-89.

Recuperado

de http://www.scielo.br/pdf/er/n31/n31a06.

- Souza Costa, K. \& Freitas, G. F. (2009). Perfil dos homens formandos na Escola de Enfermagem da Universidade de São Paulo (1950-1990). Cultura de los Cuidados, 13(26), 38-48. Recuperado de https://rua.ua.es/dspace/bitstream/10045/13512/1/ CC 26 07.pdf.
- Teixeira, E., Vale, E. G., Fernandes, J. D. \& Sordi, M. R. G. (2006). Enfermagem. Em A trajetória dos cursos de graduação na área da saúde: 1991-2004 (pp. 141-68). Brasilia: Instituto Nacional de Estudos e Pesquisas Educacionais.

- Teixeira, E., Fernandes, J. D., Andrade, A. C., Silva, K. L., Rocha, M. E. M. O. \& Lima, R. J. O. (2013). Panorama dos cursos de graduação em enfermagem no Brasil na década das Diretrizes Curriculares Nacionais. Rev. bras. Enferm, 66(spe), 102-10. Recuperado de http://www.scielo.br/pdf/reben/v66nspe/v66nspea 14.pdf.

- Wetterich, N. C., Melo, M. R. A. (2007). Perfil sociodemográfico do aluno do curso de graduação em enfermagem. Rev Latino-am Enfermagem. 15(3). 